\title{
BMJ Open Effects of a training programme of functional electrical stimulation (FES) powered cycling, recreational cycling and goal-directed exercise training on children with cerebral palsy: a randomised controlled trial protocol
}

\author{
Ellen L Armstrong, ${ }^{1,2}$ Roslyn N Boyd, ${ }^{3}$ Megan J Kentish, ${ }^{2}$ Christopher P Carty, ${ }^{1,2,4,5}$ \\ Sean A Horan ${ }^{1,4}$
}

To cite: Armstrong EL, Boyd RN Kentish MJ, et al. Effects of a training programme of functional electrical stimulation (FES) powered cycling, recreational cycling and goaldirected exercise training on children with cerebral palsy: a randomised controlled trial protocol. BMJ Open 2019;9:e024881. doi:10.1136/ bmjopen-2018-024881

- Prepublication history and additional material for this paper are available online. To view these files, please visit the journal online (http://dx.doi. org/10.1136/bmjopen-2018024881).

Received 5 July 2018

Revised 3 April 2019

Accepted 1 May 2019

A) Check for updates

(C) Author(s) (or their employer(s)) 2019. Re-use permitted under CC BY-NC. No commercial re-use. See rights and permissions. Published by BMJ.

For numbered affiliations see end of article.

Correspondence to

Ellen L Armstrong;

ellen.armstrong@griffithuni. edu.au

\section{ABSTRACT}

Introduction Children with cerebral palsy (CP) experience declines in gross motor ability as they transition from childhood to adolescence, which can result in the loss of ability to perform sit-to-stand transfers, ambulate or participate in leisure activities such as cycling. Functional electrical stimulation (FES) cycling is a novel technology that may provide opportunities for children with $\mathrm{CP}$ to strengthen their lower limbs, improve functional independence and increase physical activity participation. The proposed randomised controlled trial will test the efficacy of a training package of FES cycling, adapted cycling and goal-directed functional training to usual care in children with $\mathrm{CP}$ who are susceptible to functional declines.

Methods and analysis Forty children with CP (20 per group), aged 6-8 years and classified as Gross Motor Function Classification System (GMFCS) levels II-IV will be recruited across South East Queensland. Participants will be randomised to either an immediate intervention group, who will undertake 8 weeks of training, or a waitlist control group. The training group will attend two 1 hour sessions per week with a physiotherapist, consisting of FES cycling and goal-directed, functional exercises and a 1 hour home exercise programme per week, consisting of recreational cycling. Primary outcomes will be the gross motor function measure and Canadian occupational performance measure, and secondary outcomes will include the five times sit-tostand test, habitual physical activity (accelerometry), power output during cycling and Participation and Environment Measure-Children and Youth. Outcomes will be assessed at baseline, postintervention (8 weeks) and 8 weeks following the intervention (retention).

Ethics and dissemination Ethical approval has been obtained from Griffith University (2018/037) and the Children's Health Queensland Hospital and Health Service (CHQHHS) Human Research Ethics Committee (HREC/17/ $\mathrm{QRCH} / 88$ ). Site-specific approval was obtained from CHQHHS research governance (SSA/17/QRCH/145). Results from this trial will be disseminated via publication in relevant peer-reviewed journals.
Strengths and limitations of this study

- To our knowledge, this will be the first randomised controlled trial to investigate the efficacy of a physiotherapy intervention that combines functional electrical stimulation (FES) cycling in a clinic with recreational cycling in the community, for children with cerebral palsy (CP).

- The study will investigate whether improvements in gross motor function and goal performance and satisfaction translates to increased levels of habitual physical activity (HPA), the performance of everyday activities and participation for children with $\mathrm{CP}$, following 8 weeks of a combined physiotherapy intervention.

- Innovative, machine learning approaches will be employed to inform the optimal placement of accelerometers to detect HPA in children with CP who use walking aids or wheelchairs.

- Goal-directed and individualised exercise programmes may be challenging to replicate systematically in future FES cycling studies.

- Limited access to adapted bikes and necessary attachments in Australia may present challenges when tailoring bikes to participants who require extensive equipment modifications.

Trial registration number ACTRN12617000644369p.

\section{INTRODUCTION}

There is a paucity of evidence for interventions to improve gross motor function in children with cerebral palsy (CP) who are marginal or non-ambulant. ${ }^{2}$ This is concerning as children classified as Gross Motor Function Classification System (GMFCS) levels III-V experience a steady decline in gross motor function as they transition from childhood to 
adolescence, leading to a loss of ability to independently ambulate and self-transfer. ${ }^{3}{ }^{4} \mathrm{~A}$ decline in gross motor function can have a profound impact on families if they are required to purchase assistive devices, make modifications to vehicles or forfeit paid employment to provide full-time care. ${ }^{56}$ Access to leisure activities, transport and community-based services become increasingly challenging as gross motor function declines. This randomised controlled trial (RCT) will address a major problem in $\mathrm{CP}$, by testing the efficacy of a training package of functional electrical stimulation (FES) cycling, goal-directed functional training and recreational cycling to improve gross motor function, sit-to-stand (STS) performance and habitual physical activity (HPA) in children with CP (GMFCS II-IV). In preparation for a large multisite trial, this study will also establish the validity of activity monitors to detect HPA in children with CP who use wheelchairs or walking aids.

Training for improved gross motor function can be challenging for youth with impaired mobility due to a lack of appropriately tailored programmes and adapted exercise equipment. Cycling using stationary or adapted bikes is a logical and enjoyable means to improve exercise capacity and function in children with CP because it does not require high levels of balance and coordination compared with traditional gym-based programmes. Despite its popularity in therapy programmes for children with CP, few studies have investigated the effects of cycling on gross motor function. ${ }^{7}$ Of the limited studies available, significant, positive improvements were reported on the standing domain of the gross motor function measure (GMFM) for non-ambulant children following two stationary cycling interventions, delivered over three sessions per week for 6 weeks. ${ }^{8}$ Others have reported improved hamstring muscle strength and balance following interventions that incorporated resistance training as an adjunct to cycling training. ${ }^{11}$

Children with CP who participated in adapted cycling programmes reported that cycling allows them to 'go fast', keep up with friends and participate in family outings. ${ }^{12}$ Adapted cycling refers to cycling on a bicycle or tricycle that has been modified to accommodate the needs of the rider. Common adaptations include backrests or chest supports for stability, foot straps and adapted handlebars. Some children, however, still lack the muscle strength, coordination or selective motor control to volitionally pedal or to achieve a sufficient workload for training effects. Even if children with CP can volitionally pedal, there are key differences in lower limb alignment and muscular control compared with typically developing peers, suggesting that further adaptations may be necessary to enable participation in cycling. ${ }^{13-15}$

FES cycling provides a solution for children with CP who have limited ability to pedal due to muscle weakness, spasticity and deconditioning. ${ }^{13} 1516$ FES refers to electrical impulses that are delivered to the muscles via surface electrodes at an intensity, that is, sufficient to evoke muscular contractions. ${ }^{17}$ When applied during cycling, FES can provide sensory cues to help children contract their muscles at the correct point in the cycling phase, with the aim to improve the timing and synergy of contractions. ${ }^{13}$ Alternatively, it can evoke stronger muscle contractions to help the child achieve full cycling revolutions. $^{13} 18$

Pilot research on adolescents with $\mathrm{CP}$ have demonstrated that FES cycling can lead to immediate improvements in cycling performance such as increased cadence, power output (PO) and heart rate, ${ }^{18} 19$ as well as improved lower limb strength, oxygen expenditure and lower limb cocontraction after training for $30 \mathrm{~min}$, 3 days per week for 7 weeks. ${ }^{16}{ }^{20}$ The preliminary data support feasibility for participants to generate higher POs and reach higher training intensities to result in training effects. ${ }^{18} 1921$ It is unknown if increases in force-generating capacity from FES cycling translate to improved gross motor function or performance of non-cycling related activities.

There is evidence that strength training can improve muscle strength in children and young adults with CP; however, there is conflicting evidence on its efficacy to improve functional outcomes. ${ }^{22-26}$ Despite following progressive resistance training principles, one well-designed RCT reported improvements in strength but not walking function after completing circuit exercises three times a week for 12 weeks. ${ }^{227}$ The authors attributed the lack of functional improvement to a lack of specificity to participant's goals and suggested an individualised, goal-directed approach to strength training. ${ }^{22}$ Indeed, goal-directed approaches have been incorporated effectively into intensive exercise interventions for children with CP. ${ }^{25} 28$ Goal-directed training has therefore been incorporated into the present protocol to ensure that participants experience the health benefits of cycling as well as improvements in functional tasks that are of lifelong importance to children classified as GMFCS levels II-IV.

A key feature of goal-directed approaches is that training goals are developed in collaboration with the child and their parent and are used to drive the content of the intervention. ${ }^{28}$ In general, the literature on strength training and cycling training in children with $\mathrm{CP}$ has predominantly focused on children who are ambulant (GMFCS levels I- II) whose mobility and activity goals differ from children who ambulate with walkers or manual wheelchairs. For example, a mobility goal for a child classified as GMFCS level II may be to increase running speed or reduce falls, while a child classified as GMFCS III or IV may wish to increase upper limb strength to improve wheelchair propulsion. A goal-directed approach will ensure that individual participants are able to practice tasks that are meaningful and relevant, despite their level of gross motor function. In addition to goal-directed activities, some variation of a STS and step up activity will be incorporated into the programme for all participants, as these tasks are important to maintain independence in activities of daily living, such as transferring to and from 
chairs or wheelchairs, toileting and getting in and out of a car..$^{30} 31$

This study will deliver an original training programme that incorporates goal-oriented exercises with a programme of FES cycling and recreational cycling. Participants will be engaged in setting meaningful training goals, which will shape the content of their individualised training programme, and context-specific practice will be encouraged through a home-training component. The individualised programme was developed on the assumption that tailoring training activities to children's specific goals and incrementing the exercises using the training principles of overload and specificity may lead to the attainment of mobility, transfer and cycling goals in children with CP who are classified as GMFCS levels II-IV.

A major challenge in CP research is selecting outcome measures that are valid, reliable and sensitive to change across GMFCS levels. ${ }^{1}$ The GMFM is the best available tool to assess gross motor function in children with CP; however, it is prone to ceiling effects in independently ambulant children and is less sensitive to changes in those with more severe motor impairments. ${ }^{32}{ }^{33}$ To provide a comprehensive and meaningful overview of function, the GMFM should be used in conjunction with other activity and participation measures. ${ }^{32}$ In this study, gross motor function will be assessed in conjunction with participant's goal performance, STS and activity capacity, cycling performance, HPA as well as participation in home, school and community activities.

Previous studies on cycling for children with CP (GMFCS levels I-V) have almost exclusively focused on stationary cycling and reported unclear evidence for the efficacy of cycling to improve functional outcomes such as muscle strength, walking speed and gross motor function. ${ }^{7}$ This study will address a substantial gap in the literature by delivering an 8-week training package of FES cycling, adapted dynamic cycling and goal-directed functional training to children with CP (GMFCS levels II-IV) with the aim to improve gross motor function, cycling ability, physical function and HPA. To our knowledge, no well-designed RCT has been conducted on FES cycling combined with recreational cycling and goal-directed functional training among children with CP whom are GMFCS II-IV.

\section{METHODS}

\section{Aims and hypotheses}

The broad aim of this randomised waitlist controlled trial is to determine the efficacy of an 8-week programme of FES cycling, goal-directed functional exercises and recreational cycling to improve gross motor function (GMFM), perceived performance and satisfaction of functional mobility and cycling-related tasks (Canadian Occupational Performance Measure (COPM) and cycling performance (PO) in children with CP (GMFCS II-IV) compared with usual care. Secondary aims are to determine the efficacy of the intervention to improve STS performance, functional activity capacity, HPA, participation in home, school and community activities; and to determine if training effects are retained at follow-up. A qualitative evaluation will independently examine engagement of children and their families in the programme.

\section{Primary hypotheses}

Compared with participants in the waitlist control group (usual care), children in the immediate intervention group will have:

1. Significantly improved gross motor function according to the GMFM-88 goal dimension and total scores and GMFM-66 immediately following the 8-week intervention (T2).

2. Greater perceived performance and satisfaction ratings (by $\geq 2$ points) for achievement of child and/or caregiver identified mobility and transfer-related functional goals identified using the Canadian Occupational Performance Measure (COPM), at T2.

3. Significantly improved cycling performance (increased peak PO) immediately following the intervention (T2).

\section{Secondary hypotheses}

Compared with children in the usual care group at T2, children/youth with CP (GMFCS II-IV) who participate in the training programme will:

1. Score significantly faster times on the five times sit-tostand test (FTSST). ${ }^{31}$

2. Have significantly higher levels of HPA and spend less time in sedentary activities (measured by accelerometers over a minimum of 2 weekdays and 2 weekend days).

3. Have greater capacity to perform functional activities as measured by the Paediatric Evaluation of Disability Index-Computer Adapted Test (PEDI-CAT).

All participants (waitlist and immediate training group) who complete the training programme will:

4. Demonstrate improved participation in the home, school and community following the intervention compared with baseline, as measured by the Participation and Environment Measure-Children and Youth (PEM-CY).

5. Have greater capacity to cycle on the RT300, demonstrated by increased cycling duration, resistance, average and peak $\mathrm{PO}$ and average pulse rates at weeks 4 and 8 compared with week 1 .

6. Have improved tolerance to electrical stimulation at weeks 4 and 8 compared with week 1 .

7. Have retained improvements on the GMFM, COPM, PEDI-CAT, FTSST, PEM-CY and HPA levels above baseline scores at 8 weeks postintervention (T3 for the immediate training group, $\mathrm{T} 4$ for the control group).

\section{Ethics}

Written and informed parent/guardian consent will be obtained prior to study enrolment by the study investigator and witnessed by a person who is not directly 
related to the study. Verbal assent will be obtained from children under the age of 12 years and written assent will be obtained from children who are 12 years and older. This trial is registered with the Australian New Zealand Clinical Trials Registry and the study protocol is reported according to the Standard Protocol Items: Recommendations for Intervention Trials statement. ${ }^{34}$ Changes to the study protocol will be communicated to the ethics committee and updated on the trial registry.

\section{Study sample and recruitment}

Children with CP who are classified as GMFCS levels IIIV, aged between 6 and 18 years and live within $100 \mathrm{~km}$ from the Queensland Children's Hospital (QCH) will be identified through a patient database at the Queensland Paediatric Rehabilitation Service (QPRS) and invited to participate via email or mail out. The study will run from December 2017 to August 2019. A follow-up phone call will be made to ensure that potential participants received the study information. The study flyer and information sheets will also be made available in treatment and waiting rooms at QPRS and through newsletters that are mailed to families who attend QPRS.

\section{Inclusion criteria}

This study will include children and adolescents with CP who at study entry are/have:

1. Aged 6-18 years.

2. Classified as functioning at GMFCS levels II, III or IV, as these groups are most likely to have functional goals that align with a STS transfer, mobility and cycling intervention.

3. Goals to improve functional mobility, cycling ability or STS, stepping transfers developed in collaboration with the child, parent and therapist.

4. Adequate range of motion (ROM) in their hips, knees and ankles to complete a full revolution of the RT300 cycle (Restorative Therapies, Baltimore) crank arm.

5. Able to understand and follow instructions for GMFM testing.

6. Able to actively engage in up to $45 \mathrm{~min}$ of physiotherapy.

7. Able to verbally or non-verbally communicate pain or discomfort.

8. Able to attend training, testing and follow-up sessions at one of our training facilities.

\section{Exclusion criteria}

1. Unable to remain in a comfortable position to use the cycling equipment for up to $30 \mathrm{~min}$.

2. Hypersensitivity to touch or unable to tolerate electrical stimulation.

3. Hip displacement that causes severe pain and would prevent the child from participating in a cycling or STS intervention.

4. Lower limb joint contracture, severe spasticity or severely reduced ROM that could limit the child's ability to complete a full cycling revolution.
5. Surgery, trauma or fractures in the preceding 12 months without medical clearance to participate in the 8-week intervention.

6. Orthopaedic surgery or serial casting scheduled during the study period.

7. Any contraindication for FES, including cardiac demand pacemakers, pregnancy or skin damage that may interfere with electrode placement.

8. Any children with known cardiovascular or pulmonary diseases that have not received medical clearance to participate in the 8-week cycling intervention.

9. Uncontrolled seizures or epilepsy.

Sample size determination (according to CONSORT guidelines) Sample size calculations have been estimated based on studies that have reported minimal clinically important differences (MCIDs) for the $\mathrm{GMFM}^{35-37}$ as well as GMFM-66 change scores reported in children with CP following (1) stationary cycling ( $\mathrm{n}=11$, GMFCS level IV and V, GMFM- 66 change $=2.94$, SD 2.8), ${ }^{9}$ (2) a goal-directed, activity-focused training programme $(\mathrm{n}=22$, GMFCS levels I-V, GMFM-66 change $=3.8$, SD $4)^{38}$ and (3) intensive upper and lower limb training, including transfer tasks $(\mathrm{n}=20$, GMFCS levels II-IV, GMFM-66 change $=3$, SD 2) ${ }^{39}$ Taking into account the proposed treatment dose of 24 hours and the severity level of participants (GMFCS levels II-IV), a mean GMFM-66 change score of 3 (100 pt scale) is proposed as the minimum difference likely to indicate a meaningful clinical improvement. A sample size of 34 participants (17 in each group) are required per group to detect a mean change score of 3 points with $80 \%$ power (two-sided test at $\mathrm{p}<0.05)$. Forty children (20 participants in each group) are required to account for any attrition.

\section{Randomisation}

Participants will be randomly allocated by the Griffith University randomisation service to the intervention group or waitlist control group using computer generated block randomisation. Participants will be stratified by individual GMFCS level (II, III, IV) to minimise confounding bias and a randomly varied block size will be used. Randomisation will occur after baseline testing is complete.

\section{Blinding}

Participants and the treating therapist will be blinded to group allocation until after baseline testing (T1). Due to the nature of the intervention, treating therapists and participants cannot be blinded to group allocation after baseline testing. The primary outcome measure (GMFM) will be video recorded and scored by a blinded physiotherapist (MK) who is trained in GMFM scoring. The assessor will also be blinded to the order in which the videos were filmed (ie, T1, T2 or T3). Qualitative interviews at the end of the training programme will be conducted by an independent interviewer. 


\section{Safety and adverse events}

To monitor adverse events, all participants and their parents will be questioned by the principal investigator at weekly therapy sessions and all adverse events will be recorded on an excel spreadsheet. Minor adverse events refer to conditions such as muscle soreness, muscular fatigue or mild injuries that do not require medical attention. Major adverse events are conditions that require medical attention, such as a fracture, and would likely result in the child discontinuing the training programme. All adverse events, regardless of their severity, will be documented and reported to a senior study advisor and if serious, escalated to the ethics committee with information reported to the child's treating physician as necessary. Risk assessments, including strategies to minimise adverse events, will be completed prior to participation in the home programme, which includes cycling on an adapted tricycle under the direct supervision of a parent or guardian. For all onsite treatments, participants will be directly supervised by a physiotherapist who is trained to deliver first aid and cardiopulmonary resuscitation. A data monitoring committee is not required as this study is not a drug-related trial.

A physical activity readiness questionnaire will be included in the screening process to allow parents to report any medical conditions or medications that may impact their child's participation in an exercise programme and/or medication that may be relevant to physical activity (PA) participation (eg, asthma inhalers).

\section{Study procedure}

This study will employ a mixed-methods design, including pilot RCT arm and a qualitative, descriptive study arm. The quantitative study arm will be conducted in accordance with the Consolidated Standards of Reporting Trials (CONSORT) guidelines (figure 1) and will explore the comparative effectiveness of the training programme and usual care. ${ }^{40}$ The waitlist design ensures that participants who are 'controls' have

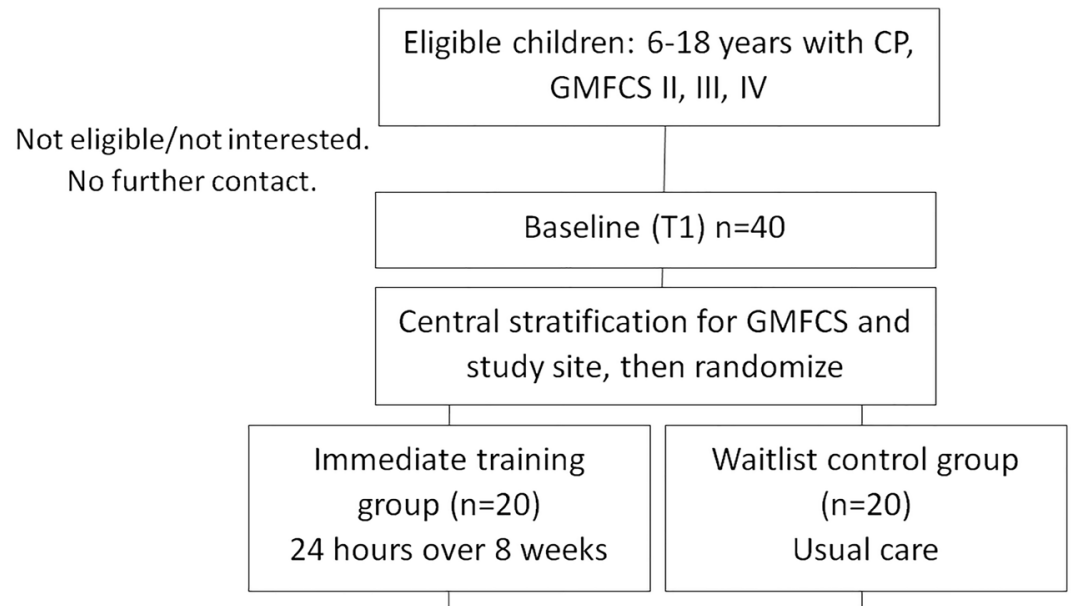

Primary outcome data collection (T2, week 10)

\begin{tabular}{|c|}
\hline $\begin{array}{c}8 \text { weeks no training } \\
\text { Usual care }\end{array}$ \\
\hline $\begin{array}{c}\text { Secondary retention } \\
\text { after } 8 \text { weeks (T3) }\end{array}$ \\
\hline
\end{tabular}

Exit week 18
Commence training

24 hours over 8 weeks

Post-intervention testing

(T3)

\begin{tabular}{|c|}
\hline $\begin{array}{c}8 \text { weeks no training } \\
\text { Usual care }\end{array}$ \\
\hline $\begin{array}{c}\text { Secondary retention } \\
\text { after } 8 \text { weeks (T4) }\end{array}$ \\
\hline
\end{tabular}

\section{Baseline Measures (T1)}

Physical activity readiness questionnaire, Parent questionnaire, GMFCS, height, weight, BMI, GMFM-88, 66 and goal total scores, COPM, FTSST, cycling peak PO, HPA (actigraphs), PA and usual care logs, PEDICAT, PEM-CY. Activity monitor trials*

\section{Assessment at week 10 (T2)}

Following 8 weeks of training Structure/function: FTSTS, cycling peak PO Activity/Participation: GMFM-88, 66 and goal total scores, COPM, PEM-CY, PEDI-CAT, PA log, HPA (triaxial accelerometers).

\section{Assessment at week 18/26 (T3/T4)}

Following 8 weeks of no-training Structure/function: FTSTS, cycling peak PO Activity/Participation: GMFM-88, 66 and goal total scores, COPM, PEM-CY, PEDI-CAT, PA log, HPA (triaxial accelerometers). Other: qualitative interview

Exit week 27

Figure 1 CONSORT flowchart demonstrating the progress of two groups through the randomised controlled trial phases. *Additional accelerometer data will be collected for a subset of 10 participants at baseline. BMI, body mass index; CONSORT, Consolidated Standards of Reporting Trials; COPM, Canadian Occupational Performance Measure; CP, cerebral palsy; FTSST, five times sit-to-stand test; HPA, habitual physical activity; PEDI-CAT, Paediatric Evaluation of Disability Index-Computer Adapted Test; PEM-CY, Participation and Environment Measure-Children and Youth; PO, power output. 
the opportunity to access the treatment immediately following the RCT period (T2).

Participants will be recruited after trial registration and ethical approval have been obtained. Potential participants will be screened against the inclusion criteria over the phone and required to attend a further screening and baseline assessment session at the QCH in Brisbane or Griffith University at the Gold Coast. Following baseline testing (T1), eligible participants will be randomised into one of two groups:

1. Intervention group: participants will immediately commence FES cycling and goal-oriented functional exercise training (two sessions per week for 8 weeks) and a goal-directed home exercise programme in addition to continuing standard care (including routine physiotherapy, occupational therapy, intramuscular botulinum toxin-A injections and so on). The same measures will be completed at baseline testing (T1, week 1) prior to commencement of the training programme and then will be repeated immediately following the 8-week training period (T2, week 10) and again following an 8-week maintenance period (T3, week 18). Participants will exit the study following T3.

2. Control group: after baseline testing and random allocation participants will continue to receive usual care (including routine physiotherapy, occupational therapy, intramuscular botulinum toxin-A injections and so on). The same measures completed at baseline testing (T1, week 1) will be repeated immediately following the waitlist control period (T2, week 10). The control group will commence training in week 11 , following T2 and outcomes will be reassessed at the end of the 8-week training period (T3, week 18). A final testing point (T4) will be completed following an 8-week maintenance period.

\section{Home visit}

At baseline, a home visit will be conducted by the primary therapist for all participants. The purpose of this visit is to (1) establish the child's and parent's specific and functional training goals, (2) identify possible barriers and facilitators to participation and strategies to improve compliance, (3) conduct an environmental screen to facilitate the development of an individualised home exercise programme that utilises existing equipment and space and (4) to provide an opportunity to trial, assess, modify and adapt cycling equipment as necessary. Goal setting will be completed using the self-care and leisure domains of the COPM and will be focused around the areas of mobility, transfers and cycling. Where possible, participants will be included in the goal setting process to ensure goals are meaningful to the individual. ${ }^{41}$ The goals established at the home visit will inform the goal-directed training component of the intervention.
Intervention group: FES cycling, recreational cycling and functional exercise training

The intervention group will complete $3 \times 60$ min training sessions per week on non-consecutive days for 8 weeks (a total of 24 sessions, total dose of 24 hours). Two sessions per week will be conducted by a qualified physiotherapist at the QPRS at the QCH, or the Menzies Health Institute Queensland at Griffith University (16 hours of direct contact over 8 weeks). The third session each week will be completed as a home programme, supervised by a parent or guardian ( 8 hours over 8 weeks). Onsite visits will consist of $30 \mathrm{~min}$ of goal-directed exercises, using a variety of functional and transfer-related tasks, followed by up to 30 min of FES cycling. The home exercise programme will consist of $30 \mathrm{~min}$ of outdoor cycling using an adapted bike and $30 \mathrm{~min}$ of functional exercises. Training days will be separated by at least 24 hours, to ensure adequate recovery time. ${ }^{42} 43$

Two onsite sessions per week were chosen instead of three, as parents of school-aged children with $\mathrm{CP}$ are known to have little free time and experience high levels of physical, emotional and financial stress. ${ }^{44}$ The weekly home exercise programme will ensure that participants achieve an adequate training dose and will reduce the time-pressures associated with travelling to a treatment facility. Further to the training programme, participants will be encouraged to increase the frequency of STS transfers at home and school to consolidate training effects and to maximise context-specific practice. Treatment adherence and PA participation will be monitored with training logs, and follow-up phone calls will be made if sessions are missed. If sessions are missed, make up sessions will be offered to optimise the minimum training dose. A target adherence of $80 \%(13 / 16)$ for onsite sessions will be set to allow for missed sessions due to unforeseen circumstances.

\section{Onsite training programme}

Onsite visits will be divided into two parts, including a goal-directed functional programme and FES cycling. Goal-directed functional exercises will be completed prior to FES cycling, as it is anticipated that fatigue following the cycling training may contribute to an increased falls risk for standing exercises. An allied health assistant or physiotherapy student will assist the principal investigator during sessions as required.

\section{Part 1: goal-directed functional training (30 mins)}

The first $30 \mathrm{~min}$ of each onsite session will consist of at least four functional exercises, two of which will be directed by participant's transfer and mobility-related COPM goals. An example COPM goal is to independently stand from a wheelchair and complete three to five steps to get into the car. Table 1 outlines an example summary of the functional exercise programme.

Training loads for the STS and step up exercises will be determined by an 8 repetition maximum (RM) STS test using a weighted vest, as a $1 \mathrm{RM}$ test may be too intense 
Table 1 Example summary of the goal-directed functional exercise programme

\begin{tabular}{|c|c|c|c|c|c|}
\hline Exercise & Load & Sets $\mathrm{x}$ reps & Rest & Progressions & Adaptations/modifications \\
\hline $\begin{array}{l}\text { 1. Loaded STS, } \\
\text { (weighted vest) }\end{array}$ & $\begin{array}{l}80 \%-100 \% 8 \mathrm{RM} \\
(\sim 60 \%-80 \% 1 \mathrm{RM})\end{array}$ & $2-3 \times 8$ & $90 \mathrm{~s}$ & $\begin{array}{l}\text { Adjusted } \\
\text { according to } \\
\text { 8RM test* }\end{array}$ & $\begin{array}{l}\text { Weighted vest will be used only if participants } \\
\text { can complete eight successful STS repetitions } \\
\text { with body weight only. Participants can use } \\
\text { assistive devices if required for safety }\end{array}$ \\
\hline $\begin{array}{l}\text { 2. Loaded step up, } \\
\text { lateral step up or } \\
\text { stairs }\end{array}$ & $\begin{array}{l}80 \%-100 \% 8 \mathrm{RM} \\
(\sim 60 \%-80 \% 1 \mathrm{RM})\end{array}$ & $2-3 \times 8$ & $90 \mathrm{~s}$ & $\begin{array}{l}\text { Adjusted } \\
\text { according to } \\
\text { 8RM test* }\end{array}$ & $\begin{array}{l}\text { Exercise will depend on the child's functional } \\
\text { ability. Body weight only if unable to complete } \\
\text { eight reps unloaded. Participants can use } \\
\text { assistive devices/support at hands if required } \\
\text { for safety. Reduce stair height if required. Part } \\
\text { task training if unable to complete the full task. }\end{array}$ \\
\hline $\begin{array}{l}\text { 3. Goal-related } \\
\text { activity } 1\end{array}$ & Unloaded & \multicolumn{3}{|c|}{$\begin{array}{l}\text { Specific to participant's goals. } \\
\text { Exact dose will be recorded. }\end{array}$} & $\begin{array}{l}\text { Activity will be adapted to be context and goal } \\
\text { specific. Part task training if unable to complete } \\
\text { full task. }\end{array}$ \\
\hline
\end{tabular}

*8RM test will be repeated twice during the 8-week programme. In between testing weeks, load will be increased by the therapist if participants can comfortably complete more than eight repetitions before reaching temporary muscle fatigue.

$\mathrm{RM}$, repetition maximum; STS, sit-to-stand.

for some children classified as GMFCS levels III and IV. ${ }^{45}$ The 8 RM STS test has been used in previous functional strength training research in children with $\mathrm{CP}$ and is thought to be equivalent to $80 \%$ of $1 \mathrm{RM}^{27}{ }^{45}$ During initial sessions, all exercises will be completed with no load to allow participants to familiarise themselves with the correct technique. ${ }^{26}$ When participants can comfortably complete 8 STS repetitions with good technique, an 8RM test protocol will be initiated to determine the training loads for subsequent sessions. As RM tests are time intensive, the $8 \mathrm{RM}$ test will be repeated twice (weeks 3 and 5) and training loads in-between testing weeks will be increased in increments of $0.25-1 \mathrm{~kg}$ if the participant can comfortably complete 8 repetitions of the exercise at the current load.

Guidelines for the predicted 8RM (loaded STS) have been established for children with ambulant CP, which are $35 \%, 30 \%$ and $25 \%$ of the body weight for GMFCS levels I, II and III, respectively. ${ }^{27} 45$ A predicted $8 \mathrm{RM}$ has not been established for GMFCS levels IV, so $20 \%$ of the body weight was chosen as a starting point for participants in this study who are GMFCS level IV. The predicted 8RM loads will be used to determine warm up loads for the actual 8RM test. As per methods described by Scholtes et $a l,{ }^{45}$ the $8 \mathrm{RM}$ test will begin with three STSs through participant's full available ROM with no load. Two additional warm-up trials of three repetitions will be completed at $50 \%$ and $70 \%$ of the predicted $8 \mathrm{RM}$ before undertaking the actual trial at $100 \%$ of the predicted $8 \mathrm{RM}$. If participants complete $<6$ or $>10$ repetitions before fatigue, weights will be removed or added to the weighted vest, ensuring that weight is evenly distributed across the front, back and sides. ${ }^{45}$ Trials will be separated by 2 min of rest.

A standardised starting position will be used, with hips and knees at $90^{\circ}$ and feet as flat as possible on the floor.
Hands will be placed on the child's lap, crossed against their chest, or on the chair arms or assistive device if normally required for a STS. To count as a successful STS, the child must move from the seated to standing position, stand for 1-2s and lower themselves into sitting without losing control (no falling) ${ }^{26}$ Flexion and extension movements during the STS task should be completed in $\sim 2-3 \mathrm{~s}$ each. ${ }^{45}$ Participants will wear normal footwear, including orthotics if these are normally worn for transfers. A detailed description of the goal-oriented functional exercise programme according to Consensus on Exercise Reporting Template (CERT) guidelines is included in online supplementary appendix 1 .

\section{Part 2: FES cycling training (30 mins)}

Following the goal-oriented functional exercise programme, participants will complete up to $30 \mathrm{~min}$ of FES cycling. Prior to randomisation, participants will have the opportunity to attend a familiarisation session to trial the equipment, get used to the sensation of FES and allow the therapist to determine the optimal starting parameters for the FES. All FES cycling training will be completed on an RT300-SLSA or RT300-SL cycle (Restorative Therapies, Baltimore), which allows users to cycle from their own wheelchair or chair (figure 2A). ${ }^{46}$

The RT300 system is designed so that the clinician selects a target speed, and the internal software adjusts the level of motor support and resistance depending on the user's ability to maintain this speed. If the user cannot maintain the target speed, motor support is automatically initiated. If the user exceeds the target speed, the level of resistance increased. Due to the expected variability in participant's cycling abilities, $\mathrm{PO}$ will be used to determine target training intensity, as PO is a product of speed and resistance and is displayed on the RT300 monitor. To 

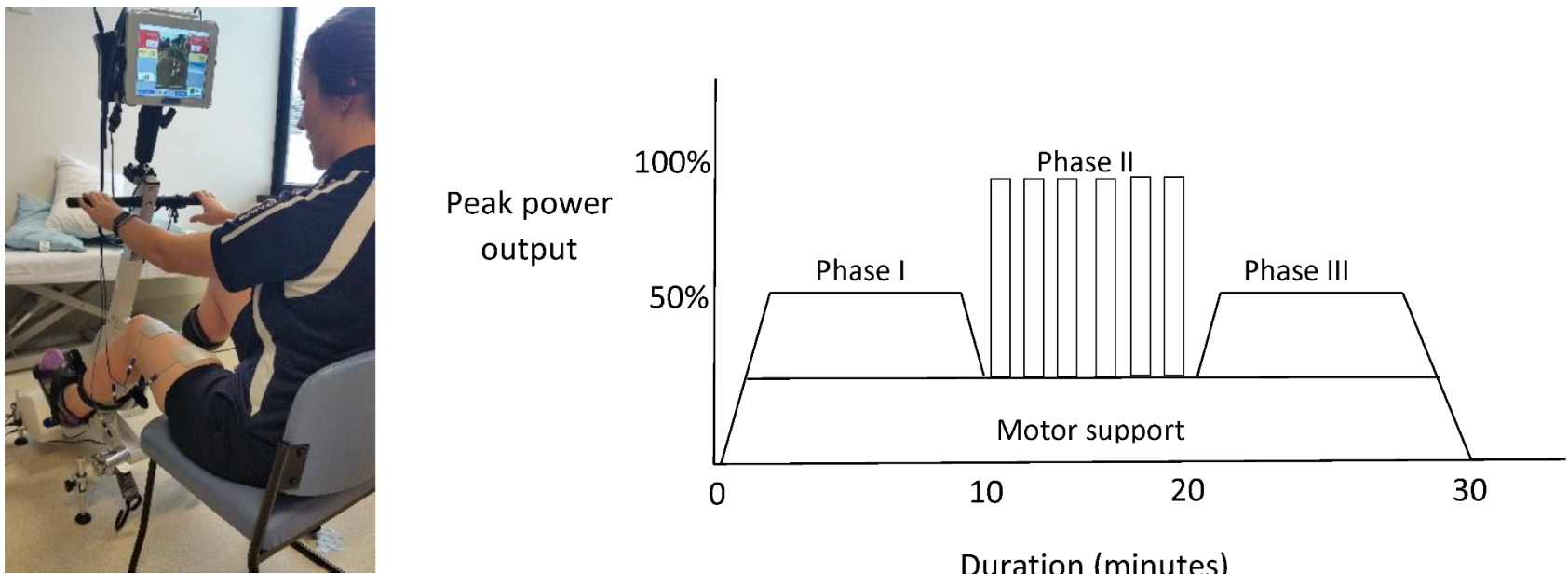

Duration (minutes)

Figure 2 (A) (left) A study investigator providing a demonstration on the RT300 FES cycle ergometer (Restorative Therapies, Baltimore). (B) (right) Graphical representation of an FES cycling session including two steady cycling phases (phase I and III) and a sprint phase (phase II). FES, functional electrical stimulation.

ensure that participants achieve a therapeutic training intensity, heart rate will be monitored by a polar M600 HR monitor. If a participant becomes visibly fatigued or severely short of breath, the session will cease until the participant feels they can recommence cycling.

Cycling sessions will be divided into three, 10-min phases (figure 2B). In phase I and III, participants will aim to cycle at $50 \%-60 \%$ of the maximal $\mathrm{PO}$ achieved during a cycling performance test. Target PO will be achieved first by increasing the speed and then by adding resistance if the child is able to cycle faster than $20 \mathrm{rpm}$. In phase II, participants will complete up to six, $30 \mathrm{~s}$ sprints at $80 \%-100 \%$ of their maximal PO, against the highest resistance achieved during phase I. Resistance will be increased further if participants exceed $45 \mathrm{rpm}$ during the sprints, as the stimulation is thought to be less effective at increasing PO above this cadence. A target heart rate for phase I and III will be $>60 \%$ of the age-predicted maximum and $>80 \%$ of the age-predicted maximum for phase II sprints. If a participant cannot complete $30 \mathrm{~min}$ of continuous cycling initially, they will be encouraged to cycle as long as they can (up to 10 mins) in phase I and III and to complete as many sprints as they can (up to 6) in phase II, with the aim to increase cycling endurance in future sessions.

To determine the target training PO each week, participants will complete $3 \times 10 \mathrm{~s}$ maximal cycling sprints. Cycling bouts will be separated by $30 \mathrm{~s}$ breaks and the average peak $\mathrm{PO}$ will be calculated. A detailed description of the cycling programme according to CERT guidelines is included in online supplementary appendix 2 .

\section{FES parameters}

Adhesive surface electrodes (PALS neurostimulation electrodes, Axelgaard, California, USA) will be applied bilaterally to the quadriceps, hamstrings, gluteal, tibialis anterior and gastrocnemius muscle groups (if tolerated) according to the manufacturer's recommendations. All participants will receive a personal set of electrodes (electrode sizes will depend on the size of participant's limbs) which will be stored in air-tight bags labelled with the patient's identification number and used for the duration of the trial.

Much of the research on FES cycling has been conducted with people with spinal cord injury (SCI) who have impaired sensation and limited or no ability to pedal volitionally. ${ }^{47}$ Children with $\mathrm{CP}$ tend to have intact or slightly reduced rather than severely impaired sensation and have the capacity to contribute some volitional effort during cycling. ${ }^{18}$ For these reasons, stimulation parameters used in training programmes for people with SCI are not appropriate for children with CP. The stimulation parameters for the FES cycling group in this study will be similar to those used in preliminary feasibility research on adolescents with CP and adjusted based on the participant's tolerance. $^{16} 1819$

A global starting frequency of $40-50 \mathrm{~Hz}$ will be used for all muscle groups. ${ }^{18}$ Fortyhertz is the default frequency on the RT300 cycle ergometer; however, Harrington found that $50 \mathrm{~Hz}$ was well tolerated by adolescents with CP. ${ }^{18} \mathrm{~A}$ frequency of $50 \mathrm{~Hz}$ falls close to the beginning of the plateau of the force-frequency curve for the quadriceps and hamstrings of children with $\mathrm{CP} .{ }^{18}{ }^{48}$ In theory, this should allow for a rapid increase in force without causing the unnecessary fatigue associated with higher frequencies that also coincide with the plateau phase. ${ }^{1848}$ For smaller muscle groups (eg, the gluteals and gastrocnemius), a frequency closer to $40 \mathrm{~Hz}$ may be more feasible.

Earlier studies on FES cycling in adolescents with CP found that an amplitude of $40 \mathrm{~mA}$ and pulse width of 90-200 $\mu$ s was well tolerated in large muscle groups (eg, quadriceps). ${ }^{18} 1949$ Based on feedback from clinicians at our treatment facility, some children tolerate a low pulse width and higher amplitude while others prefer a high pulse width and low amplitude. Pulse width and amplitude will be adjusted based on participant's tolerance, while ensuring that the overall intensity is sufficient 
to induce a motor response. If participants cannot tolerate the minimum amplitude and pulse width that are required to induce a palpable contraction, stimulation will be set to the highest tolerable level, with the aim to improve tolerance throughout the intervention.

For each training session, the FES parameters, level of motor support, cycling duration, resistance and PO will be automatically recorded on the ergometer custom software which is linked to an online database (and part of the system's usual function).

\section{Home exercise programme}

In line with the onsite training sessions, the $60 \mathrm{~min}$ home exercise programme (HEP) will be divided into $30 \mathrm{~min}$ of functional exercises and $30 \mathrm{~min}$ of adapted dynamic cycling. The functional HEP will consist of similar exercises to those practiced onsite; however, they will be supervised by a parent or guardian and performed in a context-specific environment. The cycling component will be completed using adapted tricycles or recumbent bikes at the child's home or in their community. Compliance with the HEP will be monitored with a weekly exercise diary.

The purpose of including adapted cycling in the home exercise programme is to bridge the gap between cycling in a clinical setting (FES cycling) and cycling in a functional, community-based context. Prior to randomisation, the treating physiotherapist will conduct a home visit to fit an adapted bike to the individual or to assess the participant's own equipment and organise any necessary modifications. Modifications may include simple adjustments such as changing the seat or handlebar heights, or more complex modifications such as adding trunk supports, rear-steering systems and modified foot plates. This assessment by a physiotherapist was considered a major facilitator in a previous study of adapted cycling in children with CP. ${ }^{50}$ If participants do not own their own bike and helmet, they will be provided one on loan for the duration of the study. If participants are interested in purchasing their own, the treating physiotherapist will assist the family to apply through appropriate community funding schemes. Some examples of the bikes available for loan are provided in figure 3.

Due to the variability in function between and within GMFCS levels, it is not possible to standardise the equipment used for the home cycling and exercise programme. For this reason, the exact type of bike and the modifications used for each child will be recorded in detail. The prescribed dose of cycling will be consistent, and participants will be required to record the amount of time cycled each week in a cycling diary, included in the home exercise programme.

\section{Waitlist control group: usual care}

Children in both groups will continue to receive 'usual care' for the 8-week intervention period. Usual care refers to any therapeutic treatment or service that the child would normally receive outside of the intervention study. This can vary greatly from child to child and may include no therapy at all, the occasional school-based session with a physiotherapist or occupational therapist, medical treatments such as lower limb intramuscular botulinum toxin-A injections, weekly hydrotherapy sessions or routine visits to a community-based provider such as a private physiotherapy or speech therapy clinic. A usual care $\log$ will be used to record in detail, the types and frequencies of interventions being received by all participants throughout the duration of the study. It is expected that most participants in this study will not access additional therapy on a regular basis. ${ }^{51}$

\section{Validation of activity monitors in children classified as GMFCS levels III and IV}

HPA refers to any physical activity that a person does as part of their everyday life. ${ }^{52}$ Currently, there are no accurate methods to detect HPA in children with $\mathrm{CP}$ who use wheelchairs or gait aids to mobilise. This project will employ a machine learning approach to identify patterns in the data and make predictions based on activity type (eg, walking, cycling, upper limb activity). It will also
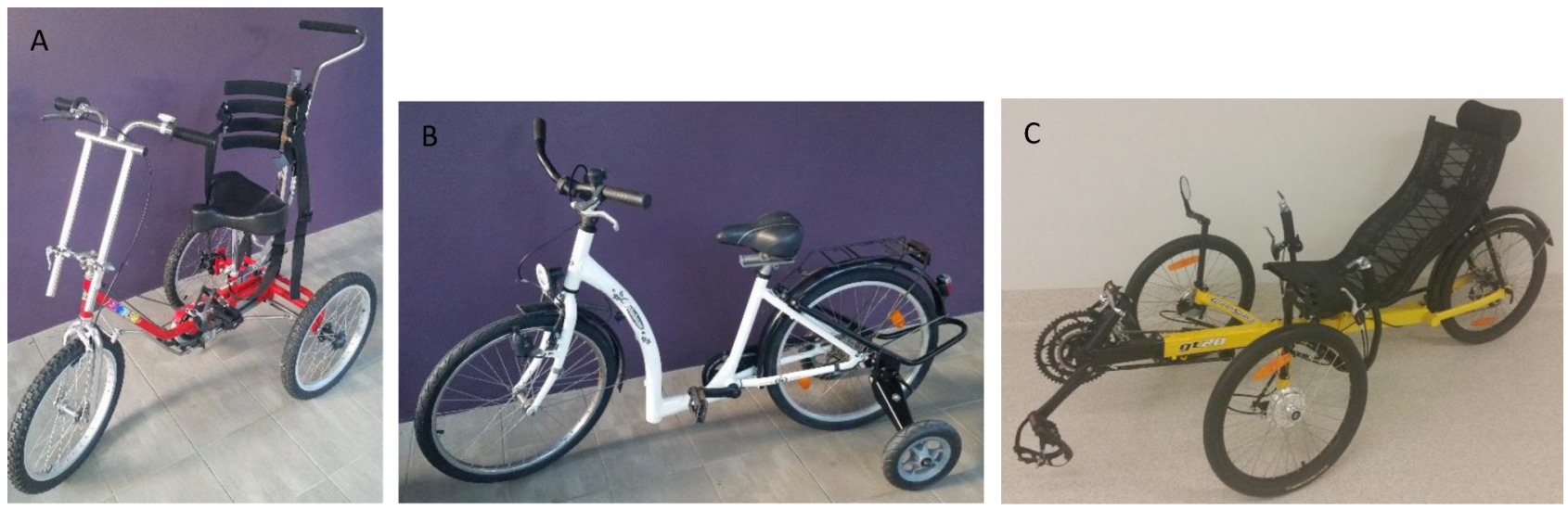

Figure 3 (A) Twenty inch upright tricycle (Body Cycles Australia, South Australia, Australia) with rear-steering attachment, back rest, pommel seat, pedal levellers and adjustable handlebars. (B) Schuchmann Momo Therapy Bike (Schuchmann GmbH \& Co. KG, Hasbergen, Germany) with large training wheels and a low frame to facilitate transfers. (C) Greenspeed GT20 recumbent trike (Greenspeed, Knoxfield, Victoria, Australia). 
determine the best placement of activity monitors to detect activities in children with $\mathrm{CP}$ who use wheelchairs or walking aids (GMFCS levels III and IV). The use of machine learning algorithms is a highly innovative approach that supersedes earlier cut-point methods that were based on activity intensity. ${ }^{52}$ Machine learning algorithms can be used to identify patterns in the accelerometer data in order to predict the activity type, which can be more informative than the activity intensity alone. Establishing the validity of activity monitors in this group of participants is necessary to accurately quantify freeliving activities in future trials of marginal or non-ambulant children with CP.

Prior to group allocation, a subset of 10 participants will complete a once-off assessment while wearing four Actigraph GT3X activity monitors (ActiGraph Corporation, Pensacola, Florida, USA). The monitors will be positioned on the dominant or least affected side, on the (1) dorsum of the wrist, (2) lateral hip, in the mid-axillary line, (3) anteriorly on the mid-thigh and (4) $1-3 \mathrm{~cm}$ superior to the lateral malleolus of the ankle. Participants will complete up to six activities, lasting 5 min each. Activities will increase in difficulty and include lying supine, sitting at a desk and colouring, throwing/catching a ball, STSs, propelling a wheelchair (if able), walking briskly (if able) and cycling. Heart rate will be monitored using a Polar M60 wrist monitor and all trials will be video recorded. Accelerometry data will be collected at a frequency of $30 \mathrm{~Hz}$ and analysed to determine the optimal placement of monitors and to investigate the validity of the ActiGraph to detect PA intensity and activity type in children classified as GMFCS levels III and IV.

\section{Training and treatment fidelity \\ Training fidelity}

The treating therapist will be a physiotherapist registered under the Australian Health Practitioner Regulation Agency. The physiotherapist will also have a formal qualification in adapted physical activity and experience working with children with disabilities in community-based and clinical settings. The therapist will be trained in the administration of the gross motor functional measure (GMFM-66) by a senior therapist who is a qualified GMFM assessor, as formal training workshops for this measure are no longer available. A qualified GMFM assessor who is blinded to treatment allocation will conduct the scoring of the GMFM assessments from video tape recordings of the testing sessions. The study therapist will also have completed a comprehensive online COPM training module in addition to being trained informally by a senior therapist.

The treating therapist will have completed all necessary online training modules for the RT300-SLSR (Restorative Therapies) in addition to attending multiple workshops and demonstrations run by an experienced physiotherapist through QPRS. Physiotherapy students or research students who assist the treating physiotherapist will be required to complete RT300 online training prior to assisting with the treatment sessions. To maximise participant's engagement in the home programme, the treating therapist will have appropriate knowledge of adapted cycling equipment, community funding for this equipment or modifications and attachments that may be necessary for participant's existing equipment. Where additional input is necessary, the treating physiotherapist will have contact with other therapists who have experience with adjusting and modifying bicycles for children with CP.

\section{Treatment fidelity}

The following strategies will be undertaken to ensure treatment fidelity:

1. Training sessions will be video recorded intermittently (with parent/caregiver consent) and reviewed by a senior author to ensure compliance with the training protocol.

2. Where possible, all sessions will be conducted by the same therapist to ensure consistency across sessions. Due to the nature of the goal-based training, the content of the functional-exercise programme may differ between participants, although efforts will be made to ensure a similar exercise dose.

3. Exercises, activities and progressions will be developed in consultation with a team of experienced paediatric physiotherapists.

4. The training programme has been developed to meet the recommended training dose (intensity, frequency, time and type) for children with $\mathrm{CP}^{42}$

5. Training and usual care logs will be completed by each participant or parent to track the home exercise training dose in addition to other training or therapeutic activities completed throughout the week.

\section{Patient and public involvement}

The research questions, design of the training programme and choice of outcome measures were directed by a literature review, with input from a therapist who had extensive experience delivering FES cycling programmes to children with neurological disorders. Patients were not directly involved in the process of study design; however, they will be involved in the goal setting process prior the training programme and will be provided opportunities to assess the burden of the intervention through a qualitative interview at study completion.

\section{Participant and data management}

The percentage of eligible participants successfully recruited, and numbers of eligible participants who choose not to participate will be recorded. Participant retention will be recorded throughout the trial period. All data will be analysed by intention-to-treat, whereby a participant's assessment from the last available time point is carried forward in the event of study withdrawal or loss to follow-up. Treatment dose is automatically recorded by the FES cycling programme and will be monitored by the therapists. Where participants are not completing the 
required dose of FES cycling, participants will be alerted and efforts will be made to increase compliance (using behaviour enhancing strategies).

Paper documents and files will be deidentified, labelled with a four-digit participant identification code and stored in a locked filing cabinet at the Centre for Children's Health Research (CCHR). Consent forms and demographic information will be kept separately, also in a locked filing cabinet at CCHR. The list of patient identification codes and all other electronic data will be stored securely through the Griffith University database.

\section{Classification measures and screening assessments}

Prior to randomisation, participant's demographic details will be collected and with permission, the patient files will be accessed to confirm a clinical diagnosis of CP, the GMFCS level (at last visit) and to screen for any conditions that may require medical clearance prior to participating in moderate to vigorous PA. The following screening and assessment tools will be completed prior to baseline testing.

Participant and family details questionnaire: this questionnaire has been developed for the purpose of this RCT and will collect important demographic details and information, that is, relevant to the intervention, such as the parent's details, known comorbidities and regular medications, access to flat, open and safe areas to cycle, whether or not the child owns a bike and so on. This tool will also screen for underlying health conditions that may exclude participants from the programme (eg, uncontrolled epilepsy) or conditions that may require medication to be on hand during training sessions.

Functional severity: the Gross Motor Function Classification System-Expanded and Revised is a valid and reliable 5-level scale used to classify children with CP aged 2-18 years according to gross motor function. ${ }^{53}$ The specific classification descriptors for each level are different depending on the age of the child; however, the broad headings range from level I (the child walks with no limitations) through to $\mathrm{V}$ (the child is transported in a manual wheelchair). Participants will be classified by the treating therapist at baseline to ensure that all classifications are up to date and to help stratify children prior to random allocation.

Motor type and distribution: the Surveillance of CP in Europe guidelines will be used to classify participant's motor type as spastic, dystonic, ataxic, hypotonic, choreoarthetosis, mixed CP or unclassifiable.$^{54}$ Distribution will be classified as unilateral or bilateral distribution and by the number of involved limbs (hemiplegia, diplegia, triplegia, quadriplegia).

Height and weight: height and weight will be recorded for all participants at baseline to identify potential confounding variables between the intervention and control groups.

FES-familiarisation session: prior to baseline assessments, potential participants will be invited to attend an FES familiarisation or trial session to screen for participant's tolerance to FES. Routine safety warnings for electrical stimulation will be given and sensation testing will be completed prior to trialling FES, which is a standard physiotherapy practice.

\section{Outcome measures}

The following outcome measures will be assessed across the WHO's International Classification of Functioning, Disability and Health (ICF) domains, including body structures and functions, activity and participation (see figure 4).

\section{Primary assessments}

1. GMFM (GMFM-88 Goal Total, GMFM-88 Total and GMFM66): the GMFM will be the primary outcome measure to detect gross motor function change in this study. ${ }^{33}$ Two versions of the GMFM are valid to assess gross motor function changes in children with $\mathrm{CP}^{3255}$ The original version (GMFM-88) is an ordinal scale, made up of five 'goal' dimensions that can be analysed separately or combined to produce a total GMFM-88 score. The five dimensions are (1) lying and rolling, (2) sitting, (3) crawling and kneeling, (4) standing and (5) walking, running and jumping. ${ }^{33}$ GMFM- 88 goal dimensions are typically chosen based on the child's age and GMFCS level and the raw scores are converted into a percentage 'goal total' score. ${ }^{33}$ All items are scored on a 4-point scale, with a higher score indicating better function. The GMFM-66 contains 66 items from the original version and is scored on an interval scale using the gross motor ability estimator software. The GMFM-66 cannot provide individual dimension scores and is therefore less sensitive to gross motor improvements in children with more severe CP (ie, GMFCS levels III-V).

GMFM-88 goal total scores are sensitive to functional changes in children with CP regardless of severity, with effect sizes exceeding 0.88 for all GMFCS groups (I/ II, III and IV/V). ${ }^{37}$ Although the GMFM- 88 goal total score correlated highly with the GMFM-66 (correlation coefficient: 0.7 ), they shared only $50 \%$ of the variance, indicating that the two measures assess slightly different aspects of gross motor function in children with CP. ${ }^{3756}$ This study will therefore report the GMFM- 88 goal total, GMFM-88 total score and GMFM-66 score to provide a more holistic picture of gross motor function in children with CP across different GMFCS levels. Gross motor goal dimensions will be chosen by the treating therapist, in collaboration with parents and child, while taking into account the participant's age and GMFCS level. All GMFM assessments will be filmed and later scored by a senior physiotherapist who is blind to group allocation and the order that assessments were filmed (T1, T2 or T3) ${ }^{57}$ All results will be reported as percentage scores.

MCIDs for the GMFM-66 and GMFM- 88 have been reported for children with $\mathrm{CP}^{35-37}$ In a study of 65 children with CP (0.5-9.4years, GMFCS levels I-V), changes in gross motor function were assessed using the GMFM-66 and GMFM- 88 over a 3 -month period. ${ }^{36}$ Participants 


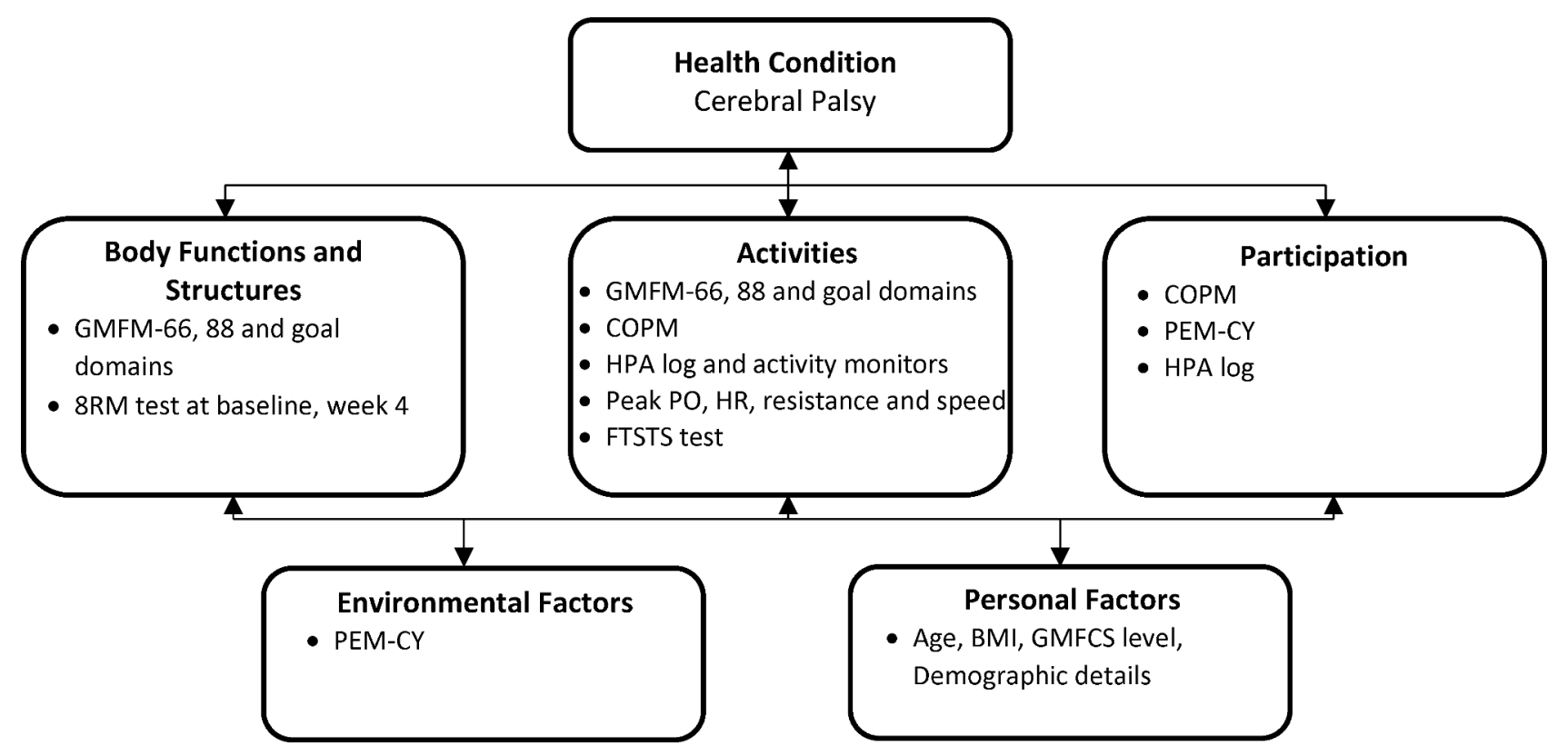

Figure 4 Study outcomes organised within an ICF framework. COPM, Canadian Occupational Performance Measure; FTSTS, five times sit-to-stand test; GMFCS, Gross Motor Function Classification System; GMFM, Gross Motor Function Measure; HPA, habitual physical activity; HR, heart rate; IFC, International Classification of Functioning, Disability and Health; PEM-CY, Participation and Environment Measure-Children and Youth; PO, power output; RM, repetition maximum.

who were perceived by their therapist to have demonstrated great improvement compared with moderate or no improvement had a mean change of 3.71 logits on the GMFM-66, and those who demonstrated a clinically meaningful improvement on the GMFM-66 compared with no improvement had a mean change of 1.58 logits.

Another study has reported the MCID for the GMFM-66 in ambulant children with CP aged 4-18 years for GMFCS levels I-III. ${ }^{35}$ The MCID for the GMFM-66 in children classified as GMFCS level III was established based on longitudinal data from 55 participants who underwent usual care without surgical intervention (with an average of 1 year and 5 months between assessments). The resulting MCIDs for this cohort were 0.7 logits for a medium effect size and 1.2 logits for a large effect (0.7). A third study reported MCID values for the GMFM- 88 goal total, GMFM-88 total and GMFM-66 based on three levels of functional severity (GMFCS I/II, III and IV/V) following 6 months of goal-directed physiotherapy ${ }^{37}$ MCIDs for the GMFM-66 were 2.37, 1.23 and 2.88 for GMFCS levels I/ II, III and IV/V, respectively. For the GMFM-88 goal total, MCIDs were 2.73, 4.35 and 4.83 and for the GMFM- 88 total, MCIDs were 3.57, 3.03 and 4.27 for GMFCS levels I/II, III and IV/V, respectively. ${ }^{37}$

2. COPM: the COPM is a client-centred measure that has been adapted for use by children to identify occupational goals across the areas of self-care (personal care, functional mobility and community management), productivity (play/school) and leisure (quiet recreation, active recreation and socialisation).$^{58}$ The COPM has demonstrated evidence of validity, reliability and clinical utility in children with CP. $^{59} 60$
The COPM will be administered during a home visit at baseline and the goals will be used to inform the content of the goal-oriented functional training programme. In collaboration with the treating therapist, participants will be asked to develop three to five transfer, mobility and/or cycling-related goals that are relevant to the self-care and leisure domains of the COPM (other domains will not be assessed in this RCT). Using a 10-point scale (1 being the lowest), participants will be asked to assign separate ratings of performance and satisfaction, to each goal. ${ }^{60}$ If children have difficulties with speech and communication, the semistructured interview will be completed by the child's parent or guardian. The COPM has been adapted for use with children, using parents and guardian's as proxies ${ }^{58}$ and has demonstrated acceptable interrater reliability when completed by parents of children with disabilities. ${ }^{61} \mathrm{~A}$ change in two points on the COPM scale is considered clinically significant. ${ }^{62}$

\section{Secondary assessments}

1. FTSST: the FTSST is a functional assessment of lower limb strength and balance and is valid for detecting improvements following task-specific training in children with CP. ${ }^{30} 3163$ It has high test-retest and inter-rater reliability and moderate concurrent validity with the Berg balance scale, timed up and go test, Paediatric Balance Scale and seated functional reach test. ${ }^{3063}$ Although it has not yet been validated for use with children classified as GMFCS level IV, it has been used to assess STS function among children with CP classified as GMFCS level II and GMFCS level III, who use assistive devices to stand ${ }^{63}$ In the absence of a gold standard measure for this group of children, the FTSST is an appropriate assessment of 
lower extremity functional strength for the intended study sample. Alternate tests such as a step up or lateral step up test are not appropriate for all children classified as GMFCS levels III and IV as they are less easily modified to accommodate lower levels of function.

The FTSST requires participants to STS five times as quickly and as safely as possible. The test will be timed from the moment the therapist says the word 'go' to the time when the participant's bottom touches the chair following the fifth repetition. This test will be repeated three times and the average time will be used as the final outcome. ${ }^{63}$ If needed, participants will be allowed to use gait aids and arm rests to achieve a STS and they will be able to rest between FTSST trials. Trials will be video recorded so that times can be checked at a later date and to ensure that the set up can be replicated during follow-up tests.

2. Cycling sprint test-peak PO: each week, participants will undertake three, 10-s sprints on the RT300 cycle ergometer (no stimulation or motor support) and the peak PO will be recorded from the ergometer's display panel. Participants will start cycling at a comfortable speed for 10-30 s and resistance will be set at the minimal level of $0.5 \mathrm{Nm}$ unless participants are able to reach a velocity of $20 \mathrm{rpm}$ or participant's feel as though their feet are flying off the pedals. Participants will then be asked to cycle as hard and as fast as possible for three $10 \mathrm{~s}$ sprints, separated by $30 \mathrm{~s}$ of passive cycling. The average peak PO from the sprints will be used to determine the target training $\mathrm{PO}$ for subsequent sessions and will also be used as a measure of functional cycling performance. In subsequent tests, the sprint resistance will be set to the highest level achieved during the previous cycling session. If participants require motor support to initiate or maintain a constant pedalling motion, the $\mathrm{PO}$ reflects the amount of work completed by the individual above the level of work completed by the motor.

3. Cycling performance measures: at the end of each cycling session, the RT300 automatically synchs data from the cycling session to RTI datalink, a secure online database. These data are only accessible to clinicians who are involved in the project who have assigned login usernames and passwords. To ensure patient confidentiality, patients are assigned a unique code and pin number prior to their first session which is used to identify cycling details in RTI datalink. To monitor cycling progress, peak and average PO, peak and average pulse rate, cycling duration, cycling distance and stimulation intensity will be recorded for each session on RTI link. Data from the last cycling session in weeks 1,4 and 8 of the training programme will be compared and analysed.

4. PEDI-CAT: the PEDI evaluates self-care, mobility and social abilities in children with CP, based on caregiver report. ${ }^{64}$ The PEDI-CAT is a refined, computer-based version of the original PEDI and is suitable for children from 0 to 21 years. ${ }^{656}$ Functional ability (activity capacity) is assessed across four domains: daily activities, mobility, social/cognitive and responsibility. The PEDI-CAT software uses statistical models to tailor each item based on responses to previous items, to minimise irrelevant questions. A scaled score is automatically generated, from 0 to 100 , with higher scores reflecting greater function. ${ }^{67}$

5. HPA levels: HPA will be recorded by triaxial accelerometers (Actigraph Corporation, Pensacola, Florida, USA) ${ }^{68}$ which are watch sized activity monitors designed to measure acceleration in three planes of movement. ActiGraph accelerometers demonstrate good concurrent validity with oxygen consumption for detecting PA intensity in adolescents with CP (GMFCS I-III) ${ }^{69} 70$ and have good to excellent reliability when HPA is monitored over consecutive days. ${ }^{69}$ Activity monitors will be fitted by a therapist and worn over seven consecutive days during the waking hours, except during water-based activities. ${ }^{68}$ A phone call, text message or email will be used to remind participants to wear the activity monitors for a minimum of 3 weekdays and 2 weekend days. Raw accelerometry data will be validated against a PA $\log$ and will be used as a measure of activity performance at the activity level of the ICF.

Accelerometry data can be collected at a pre-set frequency of $30-100 \mathrm{~Hz}$ (default is $30 \mathrm{~Hz}$ ) and at epochs as small as $1 \mathrm{~s}$. When uploaded to the Actigraph's data analysis software platform, the raw data are converted into activity counts. To gain a greater understanding of the intensity of HPA for participants, time spent in sedentary, low, moderate and vigorous PA will be determined using the most accurate GMFCS-specific cut-points at the time of data analysis. ${ }^{71}$ If an appropriate algorithm can be determined to distinguish between activity types (eg, upper limb tasks vs transfers vs cycling), the data on activity type will also be reported. The number of days worn, wear time, active versus sedentary activity, intensity of HPA and activity types (if algorithm is available) will be reported. Accelerometry data will be collected at a frequency of $30 \mathrm{~Hz}$ and accompanied by a 7-day activity $\log$.

6. Physical activity and training log: all participants will be required to complete a $\mathrm{PA}$ and training log, developed for this RCT, as a measure of training compliance and to assist with the interpretation of Actigraph data. Data from the training logs will also enable the primary investigator to calculate total training dose for each participant and the end of the trial. Participants or parents will be asked to maintain a detailed record of PA participation outside of the intervention as well as recording the total sets, repetitions and time spent completing the home exercise programme. For participants in the training group, a printed list of individualised exercises from the participant's home exercise programme will be included in the log book, with designated spaces to easily record the number of sets and reps completed and the total training duration.

7. Usual care log: a usual care log book that has previously been used in research with children with CP will be provided for participants (or parents) to record any therapeutic activity that took place in addition to the 
new training programme. ${ }^{71}$ Therapeutic activities might include visits to a community-based allied health professional, therapy sessions that occurred at school, botulinum toxin-A injections, hydrotherapy sessions and stretching or strengthening regimes completed as part of routine therapy.

8. PEM-CY: the PEM-CY is a parent-report questionnaire that explores participation and environmental factors for children aged 5-17 years at home, school and in the community. ${ }^{72}$ For each setting, parents are rate: (1) their child's level of participation in various activities on an 8-point scale (never to daily); (2) how involved their child is when doing the activity, using a 5-point scale (minimally to very involved) and (3) whether change is desired (yes or no). If yes is checked, parents also choose one of five options to indicate the type of change they would like to see (eg, be more or less involved). For each setting, parents also respond to questions about the child's environment, including questions about physical layout, social demands, resources and strategies to promote participation. $^{72}$

9. Qualitative semistructured interview: as part of the qualitative study arm, a semistructured interview will be conducted by a senior or new graduate therapist who is not the treating therapist and have a solid understanding of the study and training protocol. This qualitative study arm is optional for participants and will be completed after the participants have finished training. The purpose of the interview will be to capture the voices of participants and/or parents of participants who took part in the training programme and to gain feedback that may help to improve the provision of similar programmes in the future. In order to assist with data analysis, all conversations will be voice recorded with parental consent. A copy of the interview questions are provided in online supplementary appendix 3 .

\section{Statistical analysis}

An experienced biostatistician will provide expert input for the data analysis. Standard principles for RCTs will be followed, using two-group comparisons on all subjects on an intention-to-treat basis. Imputation techniques will be employed to avoid any bias as a result of missing data during follow-up. The primary comparison will be after 8 weeks of training (at T2) on the GMFM between groups using general linear models, with terms included for stratification and confounding variables (eg, functional severity at baseline). Similar methods will be used to determine between group differences at 18 weeks, following an 8-week no training period. For dichotomous outcomes, comparison will be by $\chi^{2}$ tests. Where continuous data exhibit skewness not overcome by transformation, non-parametric methods (Mann-Whitney U) will be used for simple comparisons.

Accelerometry data collected from the subset of 10 participants during activity trials will be validated against the video recordings and coded for activity type. The process used to determine the optimal placement of activity monitors and best method of analysis will be similar to that published in an earlier paper. ${ }^{73}$ Raw accelerometer data for each monitor (hip, wrist, thigh and ankle) will be used to create two datasets, including an xyz data series and a vector magnitude data series (where $x, y$ and $\mathrm{z}$ accelerations are combined). The statistical analysis software ' $\mathrm{R}$ ' will be used to extract various features from the two series, such as the means, SD and IQRs of the accelerations. The two data series will then be analysed using different sampling epochs (eg, 10 and $15 \mathrm{~s}$ windows) to determine the optimal sampling time for data analysis. Four different types of machine learning algorithms, including decision tree, random forest, support vector machine and logistic regression will then be applied to each data series (xyz and vector magnitude) for each location (hip, wrist, thigh and ankle) and sampling window (15 and $10 \mathrm{~s}$ ) to generate a series of models. These models will be analysed to determine which is the most accurate to detect activity type (eg, walking, cycling, wheelchair propulsion) in children with $\mathrm{CP}$ who use wheelchairs or walking aids to mobilise. Feature fusion techniques will be used to combine data from different monitor placements (hip, wrist, thigh and ankle) to help inform the best placement and optimal number of monitors to detect physical activities in this population.

\section{Qualitative analysis}

The qualitative interviews will be transcribed, and any identifiable information such as participant and school names will be removed. Psudonyms will be used to replace participant's real names. A thematic analysis approach will be taken to interpret the qualitative data obtained during the interviews. Transcripts will be read multiple times by the principal investigator and then coded for repeating, relevant or interesting comments and feedback. Similar codes will be grouped to create larger categories or themes. For example, comments that relate specifically to participating in cycling in the community can be grouped into the theme 'community participation'. Themes will be labelled and ordered in terms of importance or relevance. A theme's importance and relevance will be determined by separate reviewers and based on factors such as the frequency of comments relating to each theme. Major themes will be compared, and the connections between them explored by the principal investigators and considered in context with the research questions and existing literature.

\section{Ethics and dissemination}

The results of this study will be disseminated via publication in academic journals and through national and international conferences targeted at healthcare professionals who work in the field of paediatric rehabilitation.

\section{DISCUSSION}

This study protocol describes a waitlist RCT study design which will test the comparative effectiveness of a new 
physiotherapy training programme and usual care. The new training programme is designed to help children with $\mathrm{CP}$ aged 6-18 years who are classified as GMFCS levels II, III and IV to improve gross motor function; STS transfer performance; improve cycling ability and increase habitual PA and participation in life activities. The intervention has been designed to meet the PA recommendations for children with $\mathrm{CP}$ and will be tailored to individual participants based on self-identified transfer, mobility and cycling goals. Outcome measures have been selected across all ICF domains to address the primary and secondary study aims and hypotheses. To our knowledge, this study will be the first to investigate the effects of an FES cycling, adapted cycling and goal-directed functional training programme on activity and participation outcomes that are relevant to children and adolescents with CP. It is hoped that findings from this research will be published and disseminated in an internationally recognised, peer-reviewed journal.

Every effort will be made to control for the training dose; however, it is not pragmatic to standardise each element of the intervention due to the variable nature of CP and goal-directed interventions. Children with different levels of function are likely to require individualised adaptations to successfully cycle, and participant's functional goals are likely to differ depending on their individual needs, level of function and personal preferences. For this reason, results will be stratified according to GMFCS level and individual training programmes will be recorded and monitored closely by the principal investigator. The home visit will allow the therapist to design programmes that are suited to the individual and their home environment in an effort to improve training compliance. Finally, the training programme combines three elements which are targeted to improve functional goals relevant to children classified as GMFCS level IIIV. A limitation with this packaged approach is that it will not be possible to differentiate between the effects of the three individual elements.

\section{Author affiliations}

${ }^{1}$ School of Allied Health Sciences, Griffith University, Gold Coast, Queensland, Australia

${ }^{2}$ Queensland Paediatric Rehabilitation Service, Children's Health Queensland Hospital and Health Service, South Brisbane, Queensland, Australia

${ }^{3}$ Queensland Cerebral Palsy and Rehabilitation Research Centre, The University of Queensland, Brisbane, Queensland, Australia

${ }^{4}$ Menzies Health Institute Queensland, Griffith University, Gold Coast, Queensland, Australia

${ }^{5}$ Gold Coast Orthopaedic Research Engineering and Education Alliance, Griffith University, Gold Coast, Queensland, Australia

Acknowledgements The authors acknowledge the support of Professor Stewart Trost, in consultation regarding the use of activity monitors and data analysis and Professor Robert Ware for his guidance regarding randomization and statistical analysis.

Collaborators Stewart G Trost; Robert Ware.

Contributors All authors meet the ICMJE criteria for authorship and made substantial contributions to the study design, drafting the manuscript and proofing the final version for submission.
Funding This study will be completed in part fulfilment of ELA's doctoral thesis, under a Griffith University Higher Degree Research Scholarship (GUHDRS). RNB is supported by an NHMRC research fellowship (1105038) and CPC is supported by an Advance Queensland Mid-Career Research Fellowship (00459/2017). An RT300 cycle ergometer will be provided on loan by Restorative Therapies (Baltimore, USA) for the duration of this trial.

Competing interests None declared.

Patient consent for publication Not required.

Ethics approval Full ethical approval for this study was obtained from the Children's Health Queensland (CHQ) Ethics committee (HREC/17/QRCH/88) and sitespecific approval was granted for both sites (SSA/17/QRCH; GU: 2018/037).

Provenance and peer review Not commissioned; externally peer reviewed.

Open access This is an open access article distributed in accordance with the Creative Commons Attribution Non Commercial (CC BY-NC 4.0) license, which permits others to distribute, remix, adapt, build upon this work non-commercially, and license their derivative works on different terms, provided the original work is properly cited, appropriate credit is given, any changes made indicated, and the use is non-commercial. See: http://creativecommons.org/licenses/by-nc/4.0/.

\section{REFERENCES}

1. Ryan JM, Cassidy EE, Noorduyn SG, et al. Exercise interventions for cerebral palsy. Cochrane Database Syst Rev 2017;6:CD011660.

2. Lucas BR, Elliott EJ, Coggan S, et al. Interventions to improve gross motor performance in children with neurodevelopmental disorders: a meta-analysis. BMC Pediatr 2016;16:193.

3. Hanna SE, Bartlett DJ, Rivard LM, et al. Reference curves for the Gross Motor Function Measure: percentiles for clinical description and tracking over time among children with cerebral palsy. Phys Ther 2008;88:596-607.

4. Hanna SE, Rosenbaum PL, Bartlett DJ, et al. Stability and decline in gross motor function among children and youth with cerebral palsy aged 2 to 21 years. Dev Med Child Neurol 2009;51:295-302.

5. Bourke-Taylor H, Cotter C, Stephan R. Young children with cerebral palsy: families self-reported equipment needs and out-of-pocket expenditure. Child Care Health Dev 2014;40:654-62.

6. Morris C, Kurinczuk JJ, Fitzpatrick R, et al. Do the abilities of children with cerebral palsy explain their activities and participation? Dev Med Child Neurol 2006;48:954-61.

7. Armstrong EL, Spencer S, Kentish MJ, et al. Efficacy of cycling interventions to improve function in children and adolescents with cerebral palsy: a systematic review and meta-analysis. Clin Rehabil 2019:026921551983758.

8. Bryant E, Pountney T, Williams $\mathrm{H}$, et al. Can a six-week exercise intervention improve gross motor function for non-ambulant children with cerebral palsy? A pilot randomized controlled trial. Clin Rehabil 2013;27:150-9.

9. Williams H, Pountney T. Effects of a static bicycling programme on the functional ability of young people with cerebral palsy who are non-ambulant. Dev Med Child Neurol 2007;49:522-7.

10. Chen $\mathrm{CL}$, Hong WH, Cheng HY, et al. Muscle strength enhancement following home-based virtual cycling training in ambulatory children with cerebral palsy. Res Dev Disabil 2012;33:1087-94.

11. Mohanty P, Meshram N, Pattnaik M. Comparision of dynamic cycling vs static cycling on endurance, balance, and walking ability of children with cerebral palsy. International Journal of Physiotherapy and Research 2015;3:1163-70.

12. Pickering D, Horrocks LM, Visser KS, et al. 'Every picture tells a story': Interviews and diaries with children with cerebral palsy about adapted cycling. J Paediatr Child Health 2013;49:1040-4.

13. Johnston TE, Prosser LA, Lee SC. Differences in pedal forces during recumbent cycling in adolescents with and without cerebral palsy. Clin Biomech 2008;23:248-51.

14. Johnston TE, Barr AE, Lee SC. Biomechanics of submaximal recumbent cycling in adolescents with and without cerebral palsy. Phys Ther 2007;87:572-85.

15. Lauer RT, Johnston TE, Smith BT, et al. Lower extremity muscle activity during cycling in adolescents with and without cerebral palsy. Clin Biomech 2008;23:442-9.

16. Trevisi E, Gualdi S, De Conti C, et al. Cycling induced by functional electrical stimulation in children affected by cerebral palsy: case report. Eur J Phys Rehabil Med 2012;48:135.

17. Peckham $\mathrm{PH}$, Knutson JS. Functional electrical stimulation for neuromuscular applications. Annu Rev Biomed Eng 2005;7:327-60. 
18. Harrington AT. The development of a functional electrical stimulation assisted cycling intervention to increase fitness and strength in children with cerebral palsy [Ph.D.]. Ann Arbor: University of Delaware, 2011.

19. Harrington AT, McRae CG, Lee SC. Evaluation of functional electrical stimulation to assist cycling in four adolescents with spastic cerebral palsy. Int J Pediatr 2012;2012:1-11.

20. Peri E, Ambrosini E, Pedrocchi A, et al. Volitional cycling augmented by functional electrical stimulation in hemiparetic adolescents: A case series study. Journal of Automatic Control 2013;21:37-42.

21. Johnston TE, Wainwright SF. Cycling with functional electrical stimulation in an adult with spastic diplegic cerebral palsy. Phys Ther 2011;91:970-82.

22. Scholtes VA, Becher JG, Comuth A, et al. Effectiveness of functional progressive resistance exercise strength training on muscle strength and mobility in children with cerebral palsy: a randomized controlled trial. Dev Med Child Neurol 2010;52:e107-e113.

23. Taylor NF, Dodd KJ, Baker RJ, et al. Progressive resistance training and mobility-related function in young people with cerebral palsy: a randomized controlled trial. Dev Med Child Neurol 2013;55:806-12.

24. McNee AE, Gough M, Morrissey MC, et al. Increases in muscle volume after plantarflexor strength training in children with spastic cerebral palsy. Dev Med Child Neurol 2009;51:429-35.

25. Aviram R, Harries N, Namourah I, et al. Effects of a group circuit progressive resistance training program compared with a treadmill training program for adolescents with cerebral palsy. Dev Neurorehabil 2017;20:347-54.

26. Liao HF, Liu YC, Liu WY, et al. Effectiveness of loaded sit-tostand resistance exercise for children with mild spastic diplegia: a randomized clinical trial. Arch Phys Med Rehabil 2007;88:25-31.

27. Scholtes VA, Becher JG, Janssen-Potten YJ, et al. Effectiveness of functional progressive resistance exercise training on walking ability in children with cerebral palsy: a randomized controlled trial. Res Dev Disabil 2012;33:181-8

28. Löwing K, Bexelius A, Brogren Carlberg E. Activity focused and goal directed therapy for children with cerebral palsy--do goals make a difference? Disabil Rehabil 2009;31:1808-16.

29. Ketelaar M, Vermeer A, Hart $\mathrm{H}$, et al. Effects of a functional therapy program on motor abilities of children with cerebral palsy. Phys Ther 2001;81:1534-45.

30. Kumban W, Amatachaya S, Emasithi A, et al. Five-times-sit-to-stand test in children with cerebral palsy: reliability and concurrent validity. NeuroRehabilitation 2013;32:9.

31. Wang TH, Liao HF, Peng YC. Reliability and validity of the fiverepetition sit-to-stand test for children with cerebral palsy. Clin Rehabil 2012;26:664-71.

32. Alotaibi M, Long T, Kennedy E, et al. The efficacy of GMFM88 and GMFM-66 to detect changes in gross motor function in children with cerebral palsy (CP): a literature review. Disabil Rehabil 2014;36:617-27.

33. Russell D, Rosenbaum P, Avery L, et al. The Gross Motor Function Measure (GMFM-66 and GMFM-88) User's Manual. London: Mac Keith Press, 2002.

34. Chan AW, Tetzlaff JM, Altman DG, et al. SPIRIT 2013 Statement: defining standard protocol items for clinical trials. Rev Panam Salud Publica 2015;38:506.

35. Oeffinger D, Bagley A, Rogers S, et al. Outcome tools used for ambulatory children with cerebral palsy: responsiveness and minimum clinically important differences. Dev Med Child Neurol 2008;50:918-25.

36. Wang HY, Yang YH. Evaluating the responsiveness of 2 versions of the gross motor function measure for children with cerebral palsy. Arch Phys Med Rehabil 2006;87:51-6.

37. Ko J. Sensitivity to functional improvements of GMFM-88, GMFM66 , and PEDI mobility scores in young children with cerebral palsy. Percept Mot Skills 2014;119:305-19.

38. Sorsdahl AB, Moe-Nilssen R, Kaale HK, et al. Change in basic motor abilities, quality of movement and everyday activities following intensive, goal-directed, activity-focused physiotherapy in a group setting for children with cerebral palsy. BMC Pediatr 2010;10:26.

39. Bleyenheuft Y, Ebner-Karestinos D, Surana B, et al. Intensive upperand lower-extremity training for children with bilateral cerebral palsy: a quasi-randomized trial. Dev Med Child Neurol 2017;59:625-33.

40. Schulz KF, Altman DG, Moher D. CONSORT Group. CONSORT 2010 statement: updated guidelines for reporting parallel group randomised trials. BMJ 2010;340:c332-702.

41. Vroland-Nordstrand $\mathrm{K}$, Eliasson $\mathrm{AC}$, Jacobsson $\mathrm{H}$, et al. Can children identify and achieve goals for intervention? A randomized trial comparing two goal-setting approaches. Dev Med Child Neurol 2016;58:589-96.
42. Verschuren O, Peterson MD, Balemans AC, et al. Exercise and physical activity recommendations for people with cerebral palsy. Dev Med Child Neurol 2016;58:798-808.

43. Garber CE, Blissmer B, Deschenes MR, et al. American College of Sports Medicine position stand. Quantity and quality of exercise for developing and maintaining cardiorespiratory, musculoskeletal, and neuromotor fitness in apparently healthy adults: guidance for prescribing exercise. Med Sci Sports Exerc 2011;43:1334-59.

44. Majnemer A, Shevell M, Law M, et al. Indicators of distress in families of children with cerebral palsy. Disabil Rehabil 2012;34:1202-7.

45. Scholtes VA, Dallmeijer AJ, Rameckers EA, et al. Lower limb strength training in children with cerebral palsy--a randomized controlled trial protocol for functional strength training based on progressive resistance exercise principles. BMC Pediatr 2008;8:41.

46. Restorative Therapies Inc. 907 S Lakewood Ave, Baltimore, MD. 21224.

47. Mayson TA, Harris SR. Functional electrical stimulation cycling in youth with spinal cord injury: A review of intervention studies. $J$ Spinal Cord Med 2014;37:266-77.

48. Stackhouse SK, Binder-Macleod SA, Lee SC. Voluntary muscle activation, contractile properties, and fatigability in children with and without cerebral palsy. Muscle Nerve 2005;31:594-601.

49. McRae CG, Johnston TE, Lauer RT, et al. Cycling for children with neuromuscular impairments using electrical stimulation-development of tricycle-based systems. Med Eng Phys 2009;31:650-9.

50. Pickering DM, Horrocks L, Visser K, et al. Adapted bikes: what children and young people with cerebral palsy told us about their participation in adapted dynamic cycling. Disabil Rehabil Assist Technol 2013;8:30-7.

51. Mitchell LE, Ziviani J, Boyd RN. A randomized controlled trial of webbased training to increase activity in children with cerebral palsy. Dev Med Child Neurol 2016:58:767-73.

52. Clanchy KM, Tweedy SM, Boyd R. Measurement of habitual physical activity performance in adolescents with cerebral palsy: a systematic review. Dev Med Child Neurol 2011;53:499-505.

53. Palisano R, Rosenbaum P, Bartlett D, et al. GMFCS Expanded and Revised. Ontario: CanChild Centre for Disability Research, McMaster University, 2007

54. Europe SoCPi. Surveillance of cerebral palsy in Europe: a collaboration of cerebral palsy surveys and registers. Dev Med Child Neurol 2000;42:816-24

55. O'Connor B, Kerr C, Shields N, et al. A systematic review of evidence-based assessment practices by allied health practitioners for children with cerebral palsy. Dev Med Child Neurol 2016;58:332-47.

56. Ketelaar M, Vermeer A, Helders PJ. Functional motor abilities of children with cerebral palsy: a systematic literature review of assessment measures. Clin Rehabil 1998;12:369-80.

57. Franki I, Van den Broeck C, De Cat J, et al. A study of whether video scoring is a reliable option for blinded scoring of the Gross Motor Function Measure-88. Clin Rehabil 2015;29:809-15.

58. Cusick A, Lannin NA, Lowe K. Adapting the Canadian Occupational Performance Measure for use in a paediatric clinical trial. Disabil Rehabil 2007;29:761-6.

59. Sakzewski L, Boyd R, Ziviani J. Clinimetric properties of participation measures for 5- to 13-year-old children with cerebral palsy: a systematic review. Dev Med Child Neurol 2007;49:232-40.

60. Carswell A, McColl MA, Baptiste S, et al. The Canadian Occupational Performance Measure: a research and clinical literature review. Can $J$ Occup Ther 2004;71:210-22.

61. Verkerk GJ, Wolf MJ, Louwers AM, et al. The reproducibility and validity of the Canadian Occupational Performance Measure in parents of children with disabilities. Clin Rehabil 2006;20:980-8.

62. Law M, Baptiste S, Carswell A, et al. Canadian Occupational Performance Measure Ottowa. Ontario: COAT publications, 1998.

63. Kumban W, Amatachaya S, Emasithi A, et al. Effects of task-specific training on functional ability in children with mild to moderate cerebral palsy. Dev Neurorehabil 2013;16:410-7.

64. Haley SM, Coster WJ, Ludlow LH, et al. Paediatric Evaluation of Disability Inventory (PEDI), Version 1.0. Boston, 1992.

65. Haley SM, Raczek AE, Coster WJ, et al. Assessing mobility in children using a computer adaptive testing version of the pediatric evaluation of disability inventory. Arch Phys Med Rehabil 2005;86:932-9.

66. Haley SM, Coster WJ, Dumas HM, et al. Accuracy and precision of the Pediatric Evaluation of Disability Inventory computer-adaptive tests (PEDI-CAT). Dev Med Child Neurol 2011;53:1100-6.

67. Damiano DL, Stanley CJ, Ohlrich L, et al. Task-Specific and Functional Effects of Speed-Focused Elliptical or MotorAssisted Cycle Training in Children With Bilateral Cerebral 
Palsy: Randomized Clinical Trial. Neurorehabil Neural Repair 2017;31:736-45.

68. Trost SG, Mclver KL, Pate RR. Conducting accelerometer-based activity assessments in field-based research. Med Sci Sports Exerc 2005;37(11 Suppl):S531-S543.

69. O'Neil ME, Fragala-Pinkham M, Lennon N, et al. Reliability and Validity of Objective Measures of Physical Activity in Youth With Cerebral Palsy Who Are Ambulatory. Phys Ther 2016;96:37-45.

70. Clanchy KM, Tweedy SM, Boyd RN, et al. Validity of accelerometry in ambulatory children and adolescents with cerebral palsy. Eur J Appl Physiol 2011;111:2951-9.
71. Reedman SE, Boyd RN, Elliott C, et al. ParticiPAte CP: a protocol of a randomised waitlist controlled trial of a motivational and behaviour change therapy intervention to increase physical activity through meaningful participation in children with cerebral palsy. BMJ Open 2017;7:e015918.

72. Coster W, Law M, Bedell G, et al. Development of the participation and environment measure for children and youth: conceptual basis. Disabil Rehabil 2012;34:238-46.

73. Chowdhury AK, Tjondronegoro D, Chandran V, et al. Physical Activity Recognition Using Posterior-Adapted Class-Based Fusion of Multiaccelerometer Data. IEEE J Biomed Health Inform 2018;22:678-85. 